\title{
Synthesis of Some New Isolated/Spiro $\beta$-Lactam and Thiazolidinone Incorporating Fused Thieno Pyrimidine Derivatives
}

\author{
N.A.A. Elkanzi* \\ *Chemistry Department, Faculty of Sciense, South Valley University, Aswan, Egypt \\ *Chemistry Department, Faculty of Science, Al-Jouf University, sakaka, K.S.A.
}

\begin{abstract}
Some new spiro $\beta$-Lactam and thiazolidinone derivatives were prepared from the reaction of $\mathbf{5}$ with different aromatic amine to give Schiff bases $6 \mathbf{a}$-c followed by cycloaddition reaction with chloroacetyl chloride and/or mercaptoacetic acid to give spiro $\beta$-Lactam derivatives 7 a-c and spiro thiazolidinone 8a-c. Also Isolated $\beta$-Lactam 11a-c and Isolated thiazolidinone were prepared by the reaction of Schiff bases 10a-c with chloroacetyl chloride and/or mercaptoacetic acid, pyrazolo 14,10a-c. Isoxazolo 16a-c, pyrimidino 17a c, pyrimidin thiono 18a-c Incorporating thieno pyrimidine 9 has been synthesized by cyclocondensation addition reaction of hydrazine hydrate, phenyl hydrazine, hydroxylamine hydrochloride, urea and thiourea
\end{abstract}

Key words: Spiro $\beta$-Lactam, spiro thiazolidinone, thienopyrimidine. Schiff base, Isolated $\beta$-Lactam.

\section{Introduction}

The derivatives of fused pyrimidinones have been the focus of great interest over many years due to the fact that many compounds containing a fused pyrimidinone ring play an important role in the biochemistry of the living $\mathrm{CeU}^{1-4}$. Pyrazolo[3,4-d]pyrimidine-4-one derivatives also have extremedly rish biological activities because of their structural similarity with purines ${ }^{5,6}$, they exhibit excellent antibacterial, antiphlogistic, and antitumor activities ${ }^{7-10}$, and they are employed in the treatment of erectile dysfunction in male animals ${ }^{11-13}$. Also $\beta$-Lactam antibiotics (e.g. ampicillin, amoxicillin) are traditionally used for the treatment of common bacterial infections in both humans and food-producing animals. B-Lactam residues in foods can result in the development of new strains of bacteria resistant to these antibiotics and in allevgic reactions $\mathrm{s}^{14,15}$.

\section{Result and Discussion}

When a mixture of 2,4 diaminozcyarcothiophene $3^{16}$ and conc. Sulfuric acid was stirred at room temperature, hydrolysis of the cyano group took place to give 2,4 diaminothiophene 3-carboxylicacid amide 4 (Scheme 1). The IR spectra of compound 4 revealed the presence of $(\mathrm{C}=\mathrm{O})$ at $1645 \mathrm{~cm}^{-1},{ }^{1} \mathrm{H}-\mathrm{NMR}$ spectra revealed the presence of $4.25\left(\mathrm{brs}, 2 \mathrm{H}, \mathrm{NH}_{2}\right), 6.65\left(\mathrm{~s}, 2 \mathrm{H}, \mathrm{CONH}_{2}\right)$ which support the structure of compound 4. Reaction of compound 4 with triethyl orthoformate under reflux temperature give the corresponding compound 5. The structure of compound 5 was estahlished by IR spectra which revealed the presence of $(\mathrm{C}=\mathrm{O})$ at $1700 \mathrm{~cm}^{-1},{ }^{1} \mathrm{H}-\mathrm{NMR}$ spectra revealed the presence of $\mathrm{NH}$ at $\delta 12.59$ which support the structure of compound 5 .

It is very important to know that the formation of Schiff bases corresponding to the newly heterocyclic compounds is the cornerstone in the synthesis of the corresponding spiro and Isolated $\beta$-Lactams and thiazolidinone compounds. The activity of the carbonyl group in compound5 render it to react with different aromatic smine to give new Schiff bases 6a-c. The structures of these newly Synthesized Schiff bases 6a-c were confirmed by their elemental analysis, IR, ${ }^{1} \mathrm{H}-\mathrm{NMR}$, and mass spectra (cf. Tables I, II). The activity of the azamethine center in compound $\mathbf{6 a - c}$ is more available than the activity of the NH group toward the addition process of chloroacetyl chloride, and this mentioned phenomena is due to the presence of $\pi$ electron, which makes the foundation of the $\delta$ positive and $\delta$ negative charge on the carbon and nitrogen atom, respectively, more easy than the presence of this phenomena on the $\mathrm{NH}$ group in which the bonding between nitrogen and hydrogen wheather strong according to the nature of this bonding which leads to decreasing of the mobility desire of the hydrogen atom of this $\mathrm{pH}$ group ${ }^{17}$. Thus compound $\mathbf{6 a}-\mathbf{c}$ reacted with chloroacetyl chloride or mercaptoacetic acid to give spiro $\beta$-Lactam and spiro thiazolidinone compound ${ }^{17} \mathbf{7 a -}$ c and 8a-c. The structures of these spiro compounds 7a-c and 8a-c were confirmed by their elemental analysis, IR, ${ }^{1} \mathrm{H}-\mathrm{NMR}$ and mass spectra (cf. Tables I, II). The formation of spiro azitidine derivatives 7a-c was suggested to proceed according to the following mechanisms. 
<smiles>[X]c1ccc(/N=c2/[nH]cnc3csc(N)c23)cc1</smiles><smiles>[X]c1cccc([N+](=O)CN=c2[nH]cnc3csc(N)c23)c1</smiles>

(a)

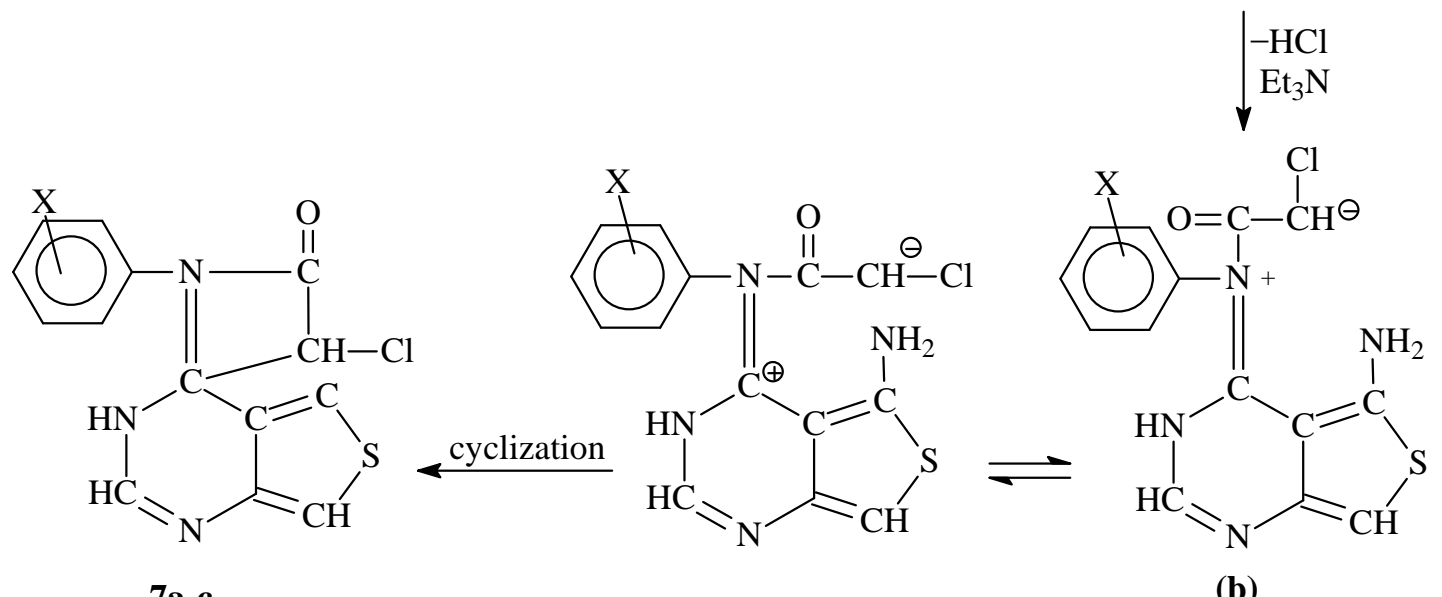

7a-c

(b)

Acetylation of thienopyrimidine $\mathbf{5}$ with one mole equivalent of acetic anhydride yielded the corresponding compound 9. The structures of compound $\mathbf{9}$ was confirmed by their elemental analysis, IR, ${ }^{1} \mathrm{H}-\mathrm{NMR}$ and mass spectra (c.f. Tables I, II).

Compound 9 react with different aromatic nitro so compound to give new Schiff bases 10a-c. The structures, of 10a-c were confirmed by their elemental analysis, IR, ${ }^{1} \mathrm{H}-\mathrm{NMR}$ and mass spectra (cf. Tables I, II). The activity of azamethine center in compound 10a-c render it available to react with chloroacetyl chloride and/or mercaptoacetic acid to give new Isolated $\beta$-Lactams and thiazolidinone compounds 11a-c and 12a-c. The structures of compounds 11a-c and 12a-c were confirmed by their elemental analysis, IR, ${ }^{1} \mathrm{H}-$ NMR and mass spectra (cf. Tables I, II). The active methyl group in the new compound $\mathbf{9}$ condensed with different aromatic aldehydes in a mixture of ethanol and DMF under piperidine as a catalyst to yield the corresponding arilidino 13a-c. The arylidino derivatives 13a-c, when interacted with hydrazine hydrate and/or phenyl hydrazine in the presence of acetic acid and/or in ethanol as solvents under piperidine as catalyst, respectively, gave the required $\mathrm{N}$-acetylpyrazolo 14a-c, and/or N-phenylpyrazolo derivatives 15a-c, respectively (Scheme 3). The arylidino derivatives 13a-c when interacted with hydroxylamine hydrochloride in a mixture of ethanol and DMF as solvent under the effect of sodium hydroxide as catalyst,gave the required isooxazolino derivatives 16a-c. The arylidino derivatives 13a-c, when interacted with urea and/or thiourea in a mixtgure of ethanol and DMF as solvent under the effect of sodium hydroxide as catalyst, gave the required pyrimidino derivatives 17a-c or, under the effect of hydrochloric acid as catalyst, gave the required pyrimidinethion derivatives $\mathbf{1 8 a - c}$.

\section{Expermental}

Melting points are uncorrected, IR spectra were measured as $\mathrm{KBr}$ pellets on a pye-unicam sp 1,000 spectrophotometer. ${ }^{1} \mathrm{H}$-NMR spectra were recorded in $\left({ }^{2} \mathrm{H}_{6}\right)$ dimethylsulfoxide at $200 \mathrm{MHz}$ on a varian Gemini NMR spectrometer using MeSi as an internal reference. Mass spectra were obtained on a Shimadzu GCMS-QP 1000 EX mass spectrometer at $70 \mathrm{eV}$. Elemental analysis were carried out at the Microanallytical center of Cairo University. 
Synthesis of 2,4-diaminothiophene 3-carboxylic acid amide 4:

Compound $3(0.139 \mathrm{~g}, 1 \mathrm{mmol})$ was added portion wise to $20 \mathrm{~mL}$ conc. Sulfuric acid, with stirring for $3 \mathrm{~h}$. The reaction mixture was poured into ice-water, neutralized, filtered, dried, and recrystallized from ethanol to give compound 4.

\section{Synthesis of compound 5:}

Compound $4(0.157 \mathrm{~g}, 1 \mathrm{mmol})$ was heated under reflux temperature in $30 \mathrm{~mL}$ formic acid for $8 \mathrm{~h}$. The reaction mixture was cooled, poured into water, filtred, dried, and the residue was recrystallized from methanol to give compound 5 (c.f. Tables I, II).

\section{Synthesis of new Schiff bases 6a-c:}

Compound $5(0.1678,1 \mathrm{mmol})$ and different aromatic amine $(1 \mathrm{mmol})$ in equindar ratios were dissolved in ethanol, and a few drops of piperidine as catalyst were added; the mixture was refluxed about 10 $\mathrm{h}$. The reaction mixture was allowed to cool at room temperature then filter, washed several times with water, dried and collected, and crystallized from the proper solvent to give 6a-c (c.f. Tables I, II).

\section{Synthesis of new spiro $\beta$-Lactam 7a-c:}

A solution of 6a-c $(1 \mathrm{mmol})$ and chloroacetyl chloride $(0.112 \mathrm{~g}, 1 \mathrm{mmol})$ in dimethyl formamide $(20$ $\mathrm{mL}$ ) in the presence of a few drops of triethylamine. The mixture was refluxed for 8-10. The filtrate was evaporated and ice-water was added, the product was separated, filtred, washed several times with water, and crystallized from the proper solvent to give 7a-c (c.f. Tables I, II).

\section{Synthesis of new piro thiazolidinone derivatives 8a-c:}

A solution of 6a-c $(1 \mathrm{mmol})$ and mercapto acetic acid $(0.0928 \mathrm{~g} 1 \mathrm{mmol})$ in dimethyl formamide (30 $\mathrm{mL}$ ) in the presence of a few drops of triethyl amine. The mixture was refluxed for 9-11 h. The filtrate was evaporated and ice. Water was added, the product was separated, filtred, washed several times with water, and crystallized from the proper solvent to give 8a-c (c.f. Tables I, II).

\section{Synthesis of compound 9:}

Compound $5(0.167 \mathrm{~g}, 1 \mathrm{mmol})$ was heated under reflux temperature in $20 \mathrm{~mL}$ glacial acetic acid/acetic anhydride (1:1) for $8 \mathrm{~h}$; then the reaction mixture was cooled, poured into water, filtred, dried, and recrystallized from ethanol to give compound $\mathbf{9}$ (c.f. Tables I, II).

\section{Synthesis of new Schiff bases 10a-c:}

Compound 9 ( $0.209 \mathrm{~g}, 1 \mathrm{mmol})$ and nitro so compound $(1 \mathrm{mmol})$ in equimolar ratios were dissolved in ethanol, and a few drops of piperidine as catalyst were added; the mixture was refluxed for 10-12 h. The reaction mixture was allowed to cool at room temperature then filtrated, washed several times with water, dried and collected, and crystallized from the proper solvent to give 10a-c (c.f. Tables I, II).

\section{Synthesis of new Isolated $\beta$-Lactam 11a-c:}

A solution of 10a-c $(1 \mathrm{mmol})$ in DMF $(30 \mathrm{~mL})$ was treated with chloroacetyl chloride $(0.112 \mathrm{gm} 1$ mmol), which was added drop by drop and stirred for $1 \mathrm{~h}$ in the presence of triethyl amine catalyst. The reaction mixture was heated under reflux for 10-12 h. (monitored by TLC). The solvent was then evaporated under reduced pressure, and the residue was treated with ice water. The solid product was collected by filtration and crystallized from the propersolvent to give 11a-c (c.f. Tables I, II).

\section{Synthesis of new Isdolated thiazolidinone derivatives 12a-c:}

A solution of 10a-c $(1 \mathrm{mmol})$ in DMF $(30 \mathrm{~mL})$ was treated with mercaptoacetic acid $(0.092 \mathrm{~g}, 1$ mmol) in the presence of triethylemine catalyst. The reaction mixture was heated under reflux for $10-12 \mathrm{~h}$. (monitored by TLC). The solvent was then evaporated under reduced pressure, and the residue was treated with ice water. The solid product was collected fy filtration and crystallized from the proper solvent to give 12a-c (c.f. Tables I, II).

\section{Synthesis of new styryle derivatives 13a-c:}

To a solution of $9(0.209 \mathrm{~g}, 1 \mathrm{mmol})$ and different aromatic aldehydes $(1 \mathrm{mmol})$ in a mixtgure of ethand and DMF as solvent and two drops of piperidine as catalyst were added. The reaction mixture was 
refluxed for 8-10 h. then left to cool and poured on cold water. The solid product so formed was collected by filtration and crystallized from the proper solvent to give 13a-c (c.f. Tables I, II).

\section{Synthesis of $\mathrm{N}$-acetyl pyrazolino derivatives 14a-c:}

To a solution of 13a-c $(1 \mathrm{mmol})$ in ethanol as solvent, hydrazine hydrate $(0.050 \mathrm{~g}, 1 \mathrm{mmol})$ was added followed by glacial acetic acid $(10 \mathrm{~mL})$ and the reaction mixture was refluxed for $(10-12) \mathrm{h}$. The reaction mixture was concentrated and coold. The residue were triturated with water, precipitates were separated, filtrated, washed several times with water and crystallized from the proper solvent to give 14a-c (c.f. Tables I, II).

\section{Synthesis of N-phenylpyrazolino derivatives 15a-c:}

To a solution of 13a-c $(1 \mathrm{mmol})$ in ethanol as solvent phenyl hydrazine $(0.108 \mathrm{~g}, 1 \mathrm{mmol})$ was added in presence of a few drops of piperidine as catalyst, and the reaction mixture was refluxed for (8-10) h. The reaction mixture was concentrated, triturated with cold water; the crystals were separated. It was filtrated, washed several times with water, and crystallized from the proper solvent to give 15a-c (c.f. Tables I, II).

\section{Synthesis of $\mathrm{N}$-isooxazolino derivatives 16a-c:}

Compound 13a-c $(1 \mathrm{mmol})$ were refluxed with hydroxylamine hydro-chloride $(0.069 \mathrm{~g}, 1 \mathrm{mmol})$ in the presence of sodium hydroxide as catalyst and ethanol as solvent for (5-6) h. The reaction mixtrure was filtrated from unreacted materials; the filtrate was triturated with cold water, the product were separated, filtrated, washed several times with water, and crystallized from the proper solvent to give 16a-c ( c.f. Tables I, II).

\section{Synthesis of N-pyrimidino derivatives 17a-c:}

Compounds 13a-c $(1 \mathrm{mmol})$ were refluxed with urea $(0.06 \mathrm{~g}, 1 \mathrm{mmol})$ in presence of $\mathrm{HCl}$ as catalyst and ethanol as solvent for (6-8) h. The reaction mixture was filtrated from unreacted materials; the filtrate was concentrated and triturated with water, the products were separated, filtrated, washed several times with water and crystallized from the proper solvent to give 17a-c (c.f. Tables I, II).

\section{Synthesis of N-thiopyrimidino derivatives 18a-c:}

Compounds 13a-c $(1 \mathrm{mmol})$ were refluxed with thiourea $(0.076 \mathrm{~g}, 1 \mathrm{mmol})$ in the presence of sodium hydroxide as catalyst and ethanol as solvent for (6-8) h. The reaction mixture was filtrated; the filtrate was concentrated, triturated with water. The products were separated, filtrated, washed several times with water and crystallized from the proper solvent to give 18a-c (c.f. Tables I, II). 
Table I

\begin{tabular}{|c|c|c|c|c|c|c|c|c|c|c|}
\hline \multirow{2}{*}{$\begin{array}{l}\text { Comp. } \\
\text { No. }\end{array}$} & \multirow{2}{*}{$\begin{array}{c}\text { Solvent of } \\
\text { Crystallization }\end{array}$} & \multirow{2}{*}{ m.p. ${ }^{\circ} \mathrm{C}$} & \multirow{2}{*}{$\begin{array}{c}\text { Yield } \\
\%\end{array}$} & \multirow{2}{*}{$\begin{array}{l}\text { M. Formula } \\
\text { (m.wt) }\end{array}$} & \multicolumn{5}{|c|}{ Analytical data found/required \% } & \multirow{2}{*}{$\begin{array}{c}\text { MS } \\
(\mathbf{m} / \mathbf{z})\end{array}$} \\
\hline & & & & & $\mathbf{C}$ & $\mathbf{H}$ & $\mathbf{N}$ & $\mathbf{S}$ & $\mathrm{Cl}$ & \\
\hline \multirow{2}{*}{4} & \multirow{2}{*}{$\mathrm{EtOH}$} & \multirow{2}{*}{275} & \multirow{2}{*}{75} & $\mathrm{C}_{5} \mathrm{H}_{7} \mathrm{ON}_{3} \mathrm{~S}$ & 38.20 & 4.49 & 26.73 & 20.40 & - & \multirow{2}{*}{157} \\
\hline & & & & $(157.18)$ & 38.19 & 4.47 & 26.72 & 20.39 & - & \\
\hline \multirow{2}{*}{5} & \multirow{2}{*}{$\mathrm{MeOH}$} & \multirow{2}{*}{290} & \multirow{2}{*}{65} & $\mathrm{C}_{6} \mathrm{H}_{5} \mathrm{ON}_{3} \mathrm{~S}$ & 43.11 & 3.01 & 25.13 & 19.18 & - & \multirow{2}{*}{167} \\
\hline & & & & (167.18) & 43.09 & 3.00 & 25.12 & 19.16 & - & \\
\hline \multirow{2}{*}{$6 a$} & \multirow{2}{*}{$\mathrm{EtOH}$} & \multirow{2}{*}{$>300$} & \multirow{2}{*}{60} & $\mathrm{C}_{12} \mathrm{H}_{10} \mathrm{ON}_{4} \mathrm{~S}$ & 59.49 & 4.16 & 23.12 & 13.23 & - & 242 \\
\hline & & & & $(242.30)$ & 59.48 & 4.15 & 23.10 & 13.21 & - & 242 \\
\hline & & & & $\mathrm{C}_{12} \mathrm{H}_{9} \mathrm{O}_{2} \mathrm{~N}_{3} \mathrm{~S}$ & 50.17 & 3.16 & 24.38 & 11.16 & - & \\
\hline $6 b$ & EtOH & $>300$ & 63 & $(287.29)$ & 50.16 & 3.14 & 24.37 & 11.14 & - & 287 \\
\hline & & 200 & & & 55.80 & 3.90 & 21.69 & 12.41 & - & \\
\hline oc & DMF/EtUH & $>300$ & $6 /$ & $\mathrm{C}_{12} \mathrm{H}_{10} \mathrm{ON}_{4} \mathrm{~S}$ & 55.78 & 3.89 & 21.67 & 12.40 & - & 258 \\
\hline & $\mathrm{DMF}$ & 200 & 61 & $\mathrm{C}_{14} \mathrm{H}_{11} \mathrm{ON}_{4} \mathrm{SCl}$ & 52.75 & 3.48 & 17.58 & 10.06 & 11.12 & \\
\hline /a & DMIF & $>300$ & 61 & (318.79) & 52.74 & 3.46 & 17.57 & 10.04 & 11.11 & 318 \\
\hline $7 \mathrm{~h}$ & $\mathrm{DMF} / \mathrm{Et} \cap \mathrm{H}$ & $>300$ & 50 & $\mathrm{C} \mathrm{H}, \mathrm{N} \mathrm{SCl}$ & 46.22 & 2.77 & 19.25 & 8.81 & 9.75 & 363 \\
\hline 70 & DMF/ETUH & $>300$ & 59 & $\mathrm{C}_{14} \mathrm{H}_{10} \mathrm{U}_{3} \mathrm{~N}_{5} \mathrm{SCl}$ & 46.21 & 2.76 & 19.23 & 8.80 & 9.73 & 503 \\
\hline & & & & $\mathrm{C}_{14} \mathrm{H}_{12} \mathrm{O}_{2} \mathrm{~N}_{4} \mathrm{SCl}$ & 50.08 & 3.60 & 16.68 & 9.55 & 10.56 & \\
\hline /c & DMF/EtUH & $>300$ & 60 & $(335.79)$ & 50.06 & 3.58 & 16.67 & 9.54 & 10.54 & 335 \\
\hline & & 200 & & $\mathrm{C}_{14} \mathrm{H}_{12} \mathrm{ON}_{4} \mathrm{~S}_{2}$ & 53.15 & 3.82 & 17.78 & 20.25 & - & \\
\hline ва & DMIF & $>300$ & 62 & (316.64) & 53.09 & 3.80 & 17.77 & 20.23 & - & 310 \\
\hline $8 \mathrm{~b}$ & $\mathrm{DMF}$ & 300 & 64 & $\mathrm{C}_{14} \mathrm{H}_{11} \mathrm{O}_{3} \mathrm{~N}_{5} \mathrm{~S}_{2}$ & 46.53 & 3.07 & 19.38 & 17.74 & - & 361 \\
\hline 80 & DIVIF & $>500$ & 04 & $(361.41)$ & 46.52 & 3.06 & 19.36 & 17.73 & - & 301 \\
\hline $8 c$ & $\mathrm{DMF}$ & $>300$ & 61 & $\mathrm{C}_{14} \mathrm{H}_{12} \mathrm{O}_{2} \mathrm{~N}_{4} \mathrm{~S}_{2}$ & 50.59 & 3.64 & 16.86 & 19.29 & - & 332 \\
\hline $8 c$ & DIVIF & $>300$ & 01 & $(332.00)$ & 50.57 & 3.63 & 16.84 & 19.28 & - & 352 \\
\hline 0 & & & & $\mathrm{C}_{8} \mathrm{H}_{17} \mathrm{O}_{2} \mathrm{~N}_{3} \mathrm{~S}$ & 45.93 & 3.37 & 20.08 & 15.32 & - & \\
\hline 9 & $\mathrm{EtOH}$ & 265 & 60 & $(209.22)$ & 45.92 & 3.35 & 20.07 & 15.31 & - & 209 \\
\hline $10 \mathrm{o}$ & MU & 200 & 60 & $\mathrm{C}_{18} \mathrm{H}_{12} \mathrm{O}_{2} \mathrm{~N}_{4} \mathrm{~S}$ & 62.06 & 3.47 & 16.08 & 9.20 & - & 210 \\
\hline IUa & MeUн & $>500$ & 02 & $(348.38)$ & 62.04 & 3.46 & 16.06 & 9.19 & - & 348 \\
\hline $10 \mathrm{~h}$ & $\mathrm{Me} \cap \mathrm{H}$ & $>300$ & 64 & $\mathrm{C}_{18} \mathrm{H}_{12} \mathrm{O}_{2} \mathrm{~N}_{4} \mathrm{~S}$ & 62.06 & 3.47 & 16.08 & 9.20 & - & 348 \\
\hline 100 & vieü & $>300$ & 04 & $(348.38)$ & 62.05 & 3.46 & 16.07 & 9.18 & - & 348 \\
\hline $10 \mathrm{c}$ & МеОH & $>300$ & 65 & $\mathrm{C}_{16} \mathrm{H}_{15} \mathrm{O}_{2} \mathrm{~N}_{5} \mathrm{~S}$ & 51.46 & 4.05 & 18.75 & 8.58 & - & 373 \\
\hline $10 \mathrm{c}$ & vieuн & $>300$ & 05 & $(373.45)$ & 51.44 & 4.04 & 18.73 & 8.57 & - & $3 / 3$ \\
\hline & & & & $\mathrm{C}_{20} \mathrm{H}_{13} \mathrm{O}_{3} \mathrm{~N}_{4} \mathrm{SCl}$ & 56.54 & 3.08 & 13.19 & 7.55 & 8.35 & \\
\hline $11 \mathrm{a}$ & EtUH & $>300$ & 61 & $(424.87)$ & 56.53 & 3.07 & 13.17 & 7.54 & 8.33 & 424 \\
\hline $11 \mathrm{~h}$ & EtOU & 200 & 62 & $\mathrm{C}_{20} \mathrm{H}_{13} \mathrm{O}_{3} \mathrm{~N}_{4} \mathrm{SCl}$ & 56.54 & 3.08 & 13.19 & 7.55 & 8.35 & 121 \\
\hline 110 & EIUH & $>300$ & 03 & $(424.87)$ & 56.52 & 3.07 & 13.18 & 7.53 & 8.33 & 424 \\
\hline $11 \mathrm{c}$ & FtOH & $>300$ & 60 & $\mathrm{C}_{18} \mathrm{H}_{16} \mathrm{O}_{3} \mathrm{~N}_{5} \mathrm{SCl}$ & 51.74 & 3.86 & 16.76 & 7.67 & 8.49 & 417 \\
\hline $11 \mathrm{c}$ & ElOH & $>500$ & OU & $(417.87)$ & 51.73 & 3.84 & 16.75 & 7.65 & 8.48 & 417 \\
\hline $12 a$ & МeOH & $>300$ & 59 & $\mathrm{C}_{20} \mathrm{H}_{14} \mathrm{O}_{3} \mathrm{~N}_{4} \mathrm{~S}_{2}$ & 56.86 & 3.34 & 13.26 & 15.18 & - & 422 \\
\hline $12 \mathrm{a}$ & vieü & $>300$ & 39 & $(422.47)$ & 56.85 & 3.32 & 13.25 & 15.16 & - & 422 \\
\hline $12 \mathrm{~b}$ & & $>300$ & & $\mathrm{C}_{20} \mathrm{H}_{14} \mathrm{O}_{3} \mathrm{~N}_{4} \mathrm{~S}_{2}$ & 56.86 & 3.34 & 13.26 & 15.18 & - & 172 \\
\hline $12 \mathrm{~b}$ & MeUH & $>300$ & 62 & $(422.47)$ & 56.84 & 3.33 & 13.24 & 15.17 & - & 422 \\
\hline 120 & $\mathrm{Med}+2)$ & & 61 & $\mathrm{C}_{18} \mathrm{H}_{17} \mathrm{O}_{3} \mathrm{~N}_{5} \mathrm{~S}_{2}$ & 52.04 & 4.12 & 16.86 & 15.43 & - & 115 \\
\hline $1 \angle \mathrm{C}$ & IveUn & $>500$ & 01 & $(415.48)$ & 52.03 & 4.10 & 16.87 & 15.42 & - & 415 \\
\hline $13 a$ & & $>300$ & 62 & $\mathrm{C}_{15} \mathrm{H}_{11} \mathrm{O}_{2} \mathrm{~N}_{5} \mathrm{~S}$ & 60.59 & 3.73 & 14.13 & 10.78 & - & \\
\hline $13 a$ & DIVI & $>500$ & 02 & (297.33) & 60.57 & 3.72 & 14.11 & 10.76 & - & 91 \\
\hline
\end{tabular}


Table 1 (Continued).

\begin{tabular}{|c|c|c|c|c|c|c|c|c|c|c|}
\hline $\begin{array}{c}\text { Comp. } \\
\text { No. }\end{array}$ & $\begin{array}{c}\text { Solvent of } \\
\text { Crystallization }\end{array}$ & m.p. ${ }^{0} \mathrm{C}$ & $\begin{array}{c}\text { Yield } \\
\%\end{array}$ & $\begin{array}{c}\text { M. Formula } \\
\text { (m.wt) }\end{array}$ & \multicolumn{5}{|c|}{ Analytical data found/required \% } & $\begin{array}{r}\text { MS } \\
(\mathbf{m} / \mathbf{z}) \\
\end{array}$ \\
\hline $13 b$ & DMF & $>300$ & 60 & $\begin{array}{c}\mathrm{C}_{15} \mathrm{H}_{11} \mathrm{O}_{3} \mathrm{~N}_{3} \mathrm{~S} \\
(313.33)\end{array}$ & $\begin{array}{l}57.50 \\
57.49\end{array}$ & $\begin{array}{l}3.54 \\
3.52\end{array}$ & $\begin{array}{l}13.41 \\
13.40\end{array}$ & $\begin{array}{l}10.23 \\
10.21\end{array}$ & - & 313 \\
\hline $13 c$ & DMF & $>300$ & 63 & $\begin{array}{c}\mathrm{C}_{15} \mathrm{H}_{10} \mathrm{O}_{4} \mathrm{~N}_{4} \mathrm{~S} \\
(342.33)\end{array}$ & $\begin{array}{l}52.63 \\
52.61\end{array}$ & $\begin{array}{l}2.94 \\
2.93\end{array}$ & $\begin{array}{l}16.37 \\
16.35\end{array}$ & $\begin{array}{l}9.37 \\
9.36\end{array}$ & - & 342 \\
\hline $14 a$ & $\mathrm{MeOH}$ & $>300$ & 60 & $\begin{array}{c}\mathrm{C}_{17} \mathrm{H}_{13} \mathrm{O}_{2} \mathrm{~N}_{5} \mathrm{~S} \\
(351.38)\end{array}$ & $\begin{array}{l}58.12 \\
58.11\end{array}$ & $\begin{array}{l}3.73 \\
3.72\end{array}$ & $\begin{array}{l}19.93 \\
19.92\end{array}$ & $\begin{array}{l}9.12 \\
9.10\end{array}$ & - & 351 \\
\hline $14 \mathrm{~b}$ & $\mathrm{MeOH}$ & $>300$ & 62 & $\begin{array}{c}\mathrm{C}_{17} \mathrm{H}_{13} \mathrm{O}_{3} \mathrm{~N}_{5} \mathrm{~S} \\
(367.38)\end{array}$ & $\begin{array}{l}55.58 \\
55.56\end{array}$ & $\begin{array}{l}3.57 \\
3.56\end{array}$ & $\begin{array}{l}19.06 \\
19.04\end{array}$ & $\begin{array}{l}8.73 \\
8.71\end{array}$ & - & 367 \\
\hline $14 c$ & $\mathrm{MeOH}$ & $>300$ & 61 & $\begin{array}{c}\mathrm{C}_{17} \mathrm{H}_{12} \mathrm{O}_{4} \mathrm{~N}_{6} \mathrm{~S} \\
(396.38)\end{array}$ & $\begin{array}{l}51.51 \\
51.50\end{array}$ & $\begin{array}{l}3.05 \\
3.03\end{array}$ & $\begin{array}{l}21.20 \\
21.19\end{array}$ & $\begin{array}{l}8.09 \\
8.07\end{array}$ & - & 396 \\
\hline $15 \mathrm{a}$ & $\mathrm{EtOH}$ & $>300$ & 59 & $\begin{array}{c}\mathrm{C}_{21} \mathrm{H}_{15} \mathrm{ON}_{5} \mathrm{~S} \\
(385.44)\end{array}$ & $\begin{array}{l}65.44 \\
65.42\end{array}$ & $\begin{array}{l}3.92 \\
3.91\end{array}$ & $\begin{array}{l}18.17 \\
18.15\end{array}$ & $\begin{array}{l}8.32 \\
8.31\end{array}$ & - & 385 \\
\hline $15 b$ & $\mathrm{EtOH}$ & $>300$ & 56 & $\begin{array}{c}\mathrm{C}_{21} \mathrm{H}_{15} \mathrm{O}_{2} \mathrm{~N}_{5} \mathrm{~S} \\
(401.44)\end{array}$ & $\begin{array}{l}62.83 \\
62.82\end{array}$ & $\begin{array}{l}3.77 \\
3.75\end{array}$ & $\begin{array}{l}17.45 \\
17.44\end{array}$ & $\begin{array}{l}7.99 \\
7.97\end{array}$ & - & 401 \\
\hline $15 c$ & $\mathrm{EtOH}$ & $>300$ & 55 & $\begin{array}{c}\mathrm{C}_{21} \mathrm{H}_{14} \mathrm{O}_{3} \mathrm{~N}_{6} \mathrm{~S} \\
(430.44)\end{array}$ & $\begin{array}{l}58.60 \\
58.59\end{array}$ & $\begin{array}{l}3.28 \\
3.27\end{array}$ & $\begin{array}{l}19.52 \\
19.50\end{array}$ & $\begin{array}{l}7.45 \\
7.44\end{array}$ & - & 430 \\
\hline $16 \mathrm{a}$ & $\mathrm{MeOH}$ & $>300$ & 54 & $\begin{array}{c}\mathrm{C}_{15} \mathrm{H}_{10} \mathrm{O}_{2} \mathrm{~N}_{4} \mathrm{~S} \\
(310.34)\end{array}$ & $\begin{array}{l}58.06 \\
58.04\end{array}$ & $\begin{array}{l}3.25 \\
3.24\end{array}$ & $\begin{array}{l}18.05 \\
18.03\end{array}$ & $\begin{array}{l}10.33 \\
10.32\end{array}$ & - & 310 \\
\hline $16 b$ & $\mathrm{MeOH}$ & $>300$ & 55 & $\begin{array}{c}\mathrm{C}_{15} \mathrm{H}_{10} \mathrm{O}_{3} \mathrm{~N}_{4} \mathrm{~S} \\
(326.33)\end{array}$ & $\begin{array}{l}55.21 \\
55.20\end{array}$ & $\begin{array}{l}3.09 \\
3.07\end{array}$ & $\begin{array}{l}17.17 \\
17.16\end{array}$ & $\begin{array}{l}9.82 \\
9.80\end{array}$ & - & 326 \\
\hline $16 \mathrm{c}$ & $\mathrm{MeOH}$ & $>300$ & 52 & $\begin{array}{c}\mathrm{C}_{15} \mathrm{H}_{9} \mathrm{O}_{4} \mathrm{~N}_{5} \mathrm{~S} \\
(355.33)\end{array}$ & $\begin{array}{l}50.70 \\
50.69\end{array}$ & $\begin{array}{l}2.55 \\
2.54\end{array}$ & $\begin{array}{l}19.71 \\
19.69\end{array}$ & $\begin{array}{l}9.02 \\
9.00\end{array}$ & - & 355 \\
\hline $17 \mathrm{a}$ & $\mathrm{EtOH}$ & $>300$ & 56 & $\begin{array}{c}\mathrm{C}_{16} \mathrm{H}_{11} \mathrm{O}_{2} \mathrm{~N}_{5} \mathrm{~S} \\
(337.35)\end{array}$ & $\begin{array}{l}56.97 \\
56.96\end{array}$ & $\begin{array}{l}3.29 \\
3.27\end{array}$ & $\begin{array}{l}4.15 \\
4.14\end{array}$ & $\begin{array}{l}9.50 \\
9.48\end{array}$ & - & 337 \\
\hline $17 b$ & DMF & $>300$ & 57 & $\begin{array}{c}\mathrm{C}_{16} \mathrm{H}_{11} \mathrm{O}_{3} \mathrm{~N}_{5} \mathrm{~S} \\
(353.35)\end{array}$ & $\begin{array}{l}54.39 \\
54.38\end{array}$ & $\begin{array}{l}3.14 \\
3.12\end{array}$ & $\begin{array}{l}19.82 \\
19.81\end{array}$ & $\begin{array}{l}9.07 \\
9.05\end{array}$ & - & 353 \\
\hline $17 \mathrm{c}$ & $\mathrm{DMF} / \mathrm{EtOH}$ & $>300$ & 54 & $\begin{array}{c}\mathrm{C}_{16} \mathrm{H}_{10} \mathrm{O}_{4} \mathrm{~N}_{6} \mathrm{~S} \\
(382.35)\end{array}$ & $\begin{array}{l}50.26 \\
50.24\end{array}$ & $\begin{array}{l}2.62 \\
2.61\end{array}$ & $\begin{array}{l}21.98 \\
21.96\end{array}$ & $\begin{array}{l}8.38 \\
8.37\end{array}$ & - & 382 \\
\hline $18 \mathrm{a}$ & $\mathrm{EtOH}$ & $>300$ & 55 & $\begin{array}{c}\mathrm{C}_{16} \mathrm{H}_{10} \mathrm{O}_{4} \mathrm{~N}_{5} \mathrm{~S}_{2} \\
(353.42)\end{array}$ & $\begin{array}{l}54.38 \\
54.37\end{array}$ & $\begin{array}{l}3.14 \\
3.12\end{array}$ & $\begin{array}{l}19.82 \\
19.81\end{array}$ & $\begin{array}{l}18.14 \\
18.12\end{array}$ & - & 353 \\
\hline $18 b$ & $\mathrm{EtOH}$ & $>300$ & 53 & $\begin{array}{c}\mathrm{C}_{16} \mathrm{H}_{11} \mathrm{O}_{2} \mathrm{~N}_{5} \mathrm{~S}_{2} \\
(369.41)\end{array}$ & $\begin{array}{l}52.02 \\
52.00\end{array}$ & $\begin{array}{l}3.00 \\
2.99\end{array}$ & $\begin{array}{l}18.96 \\
18.94\end{array}$ & $\begin{array}{l}17.36 \\
17.35\end{array}$ & - & 369 \\
\hline $18 \mathrm{c}$ & $\mathrm{EtOH}$ & $>300$ & 52 & $\begin{array}{c}\mathrm{C}_{16} \mathrm{H}_{10} \mathrm{O}_{3} \mathrm{~N}_{6} \mathrm{~S}_{2} \\
(398.41)\end{array}$ & $\begin{array}{l}48.24 \\
48.23\end{array}$ & $\begin{array}{l}2.53 \\
2.51\end{array}$ & $\begin{array}{l}21.09 \\
21.08\end{array}$ & $\begin{array}{l}16.09 \\
16.07\end{array}$ & - & 398 \\
\hline
\end{tabular}




\section{Table II}

\begin{tabular}{|c|c|c|}
\hline Compound No. & IR $v_{\max } / \mathbf{c m}^{-1}$ & ${ }^{1}$ H-NMR (DMSO) ppm \\
\hline 4 & $1645(\mathrm{C}=\mathrm{O}), 3100-3400\left(\mathrm{NH}_{2}\right)$ & $\begin{array}{l}\delta 4.25\left(\mathrm{brs}, 2 \mathrm{H}, \mathrm{NH}_{2}\right), \delta 6.65\left(\mathrm{~s}, 2 \mathrm{H}, \mathrm{CONH}_{2}\right), \\
8.00-7.01(\mathrm{~m}, 3 \mathrm{H}, \text { aromatic protons). }\end{array}$ \\
\hline 5 & $\begin{array}{l}1700(\mathrm{C}=\mathrm{O}), 3100-3400(\mathrm{NH}, \\
\left.\mathrm{NH}_{2}\right) \text {. }\end{array}$ & $\begin{array}{l}\delta 8.01-7.01(\mathrm{~m}, 5 \mathrm{H} \text {, aromatic protons), } \delta 12.59 \\
(\mathrm{br}, 1 \mathrm{H}, \mathrm{NH}) .\end{array}$ \\
\hline $6 a$ & $\begin{array}{l}1585(\mathrm{C}=\mathrm{N}), 3100-3450(\mathrm{NH}, \\
\left.\mathrm{NH}_{2}\right) .\end{array}$ & $\begin{array}{l}\delta 8.10-7.01 \text { (m, 9H, aromatic protons), } \delta 11.5 \\
\text { (brs, NH). }\end{array}$ \\
\hline $6 b$ & $\begin{array}{l}1595(\mathrm{C}=\mathrm{N}), 3100-3400(\mathrm{NH} \\
\left.\mathrm{NH}_{2}\right) .\end{array}$ & $\begin{array}{l}\delta 7.01-7.01 \text { (m, } 8 \mathrm{H} \text {, aromatic protons), } \delta 12 \\
\text { (brs, NH). }\end{array}$ \\
\hline $6 c$ & $\begin{array}{l}1590(\mathrm{C}=\mathrm{N}), 3100-3450(\mathrm{NH}, \\
\left.\mathrm{NH}_{2}, \mathrm{OH}\right)\end{array}$ & $\begin{array}{l}\delta 8.01,7.01 \text { (m, 9H aromatic protons), } \delta 10.5 \\
\text { (brs, } \mathrm{NH}) .\end{array}$ \\
\hline $7 \mathrm{a}$ & $\begin{array}{l}1650-1725(\mathrm{C}=\mathrm{O}), 3100-3400 \\
\left(\mathrm{NH}, \mathrm{NH}_{2}\right) .\end{array}$ & $\begin{array}{l}\delta 8.01-7.01 \text { (m, } 10 \text { Haromatic protons), } \delta 10.45 \\
\text { (brs, NH). }\end{array}$ \\
\hline $7 b$ & $\begin{array}{l}1655-1725(\mathrm{C}=\mathrm{O}), 3100-3400 \\
\left(\mathrm{NH}, \mathrm{NH}_{2}\right) .\end{array}$ & $\begin{array}{l}\delta 8.01-7.01 \text { (m, 9H aromatic protons), } \delta 10.85 \\
\text { (brs, NH). }\end{array}$ \\
\hline $7 \mathrm{c}$ & $\begin{array}{l}1655-1720(\mathrm{C}=\mathrm{O}), 3100-3450 \\
\left(\mathrm{NH}, \mathrm{NH}_{2}, \mathrm{OH}\right) .\end{array}$ & $\begin{array}{l}\delta 8.01-7.01 \text { (m, } 11 \mathrm{H} \text { aromatic protons), } 10.99 \\
\text { (brs, NH). }\end{array}$ \\
\hline $8 \mathrm{a}$ & $\begin{array}{l}1650-1717(\mathrm{C}=\mathrm{O}), 3100-3400 \\
\left(\mathrm{NH}, \mathrm{NH}_{2}\right) .\end{array}$ & $\begin{array}{l}\delta 2.5\left(\mathrm{CH}_{2} \text { of thiazolidinone), } \delta 8.01-7.01(\mathrm{~m}, 9\right. \\
\text { Haromatic protons }), \delta 10.35 \text { (brs, } \mathrm{NH}) .\end{array}$ \\
\hline $8 b$ & $\begin{array}{l}1660-1720(\mathrm{C}=\mathrm{O}), 3100-3400 \\
\left(\mathrm{NH}, \mathrm{NH}_{2}\right) .\end{array}$ & $\begin{array}{l}\delta 2.5\left(\mathrm{CH}_{2} \text { of thiazolidinone }\right), \delta 8.01-7.01(\mathrm{~m}, 8 \\
\text { Haromatic protons }), \delta 10.6(\mathrm{brs}, \mathrm{NH}) .\end{array}$ \\
\hline $8 \mathrm{c}$ & $\begin{array}{l}1655-1725(\mathrm{C}=\mathrm{O}), 3100-3450 \\
\left(\mathrm{NH}, \mathrm{NH}_{2}\right) .\end{array}$ & $\begin{array}{l}\delta 2.5\left(\mathrm{CH}_{2} \text { of thiazolidinone), } \delta 8.01-7.01(\mathrm{~m}, 9\right. \\
\text { Haromatic protons), } \delta 10.45 \text { (brs, } \mathrm{NH}) .\end{array}$ \\
\hline 9 & $\begin{array}{l}1705(\mathrm{C}=\mathrm{O}), 1650(\mathrm{C}=\mathrm{O}), \\
3100-3450\left(\mathrm{NH}_{2}\right) .\end{array}$ & $\begin{array}{l}\delta 2.15\left(\mathrm{~s}, 3 \mathrm{H}, \mathrm{CH}_{3}\right), \delta 8.01-7.01(\mathrm{~m}, 4 \mathrm{H} \\
\text { aromatic protons). }\end{array}$ \\
\hline $10 \mathrm{a}$ & $\begin{array}{l}1580(\mathrm{C}=\mathrm{N}), 1650-1725 \\
(2 \mathrm{C}=\mathrm{O}), 3100-3450\left(\mathrm{NH}_{2}, \mathrm{OH}\right) .\end{array}$ & $\begin{array}{l}\left.\delta 6.79 \text { (brs, } 2 \mathrm{H}, \mathrm{NH}_{2}\right), \delta 8.01-7.01(\mathrm{~m}, 10 \mathrm{H} \\
\text { aromatic protons). }\end{array}$ \\
\hline $10 \mathrm{~b}$ & $\begin{array}{l}1585(\mathrm{C}=\mathrm{N}), 1655-1720 \\
(2 \mathrm{C}=\mathrm{O}), 3100-3450\left(\mathrm{NH}_{2}, \mathrm{OH}\right) .\end{array}$ & $\begin{array}{l}\left.\delta 6.59 \text { (brs, } 2 \mathrm{H}, \mathrm{NH}_{2}\right), \delta 8.1-7.01(\mathrm{~m}, 10 \mathrm{H} \\
\text { aromatic protons). }\end{array}$ \\
\hline $10 \mathrm{c}$ & $\begin{array}{l}1590(\mathrm{C}=\mathrm{N}), 1650-1715 \\
(2 \mathrm{C}=\mathrm{O}), 3100-3400\left(\mathrm{NH}_{2}\right) .\end{array}$ & $\begin{array}{l}\delta 1.21\left(\mathrm{~s}, 6 \mathrm{H}, 2 \mathrm{CH}_{3}\right), \delta 6.12\left(\mathrm{brs}, 2 \mathrm{H}, \mathrm{NH}_{2}\right), 8.1- \\
7.01(\mathrm{~m}, 7 \mathrm{H} \text { aromatic protons). }\end{array}$ \\
\hline $11 \mathrm{a}$ & $\begin{array}{l}1650-1720(3 \mathrm{C}=\mathrm{O}), 3100-3450 \\
\left(\mathrm{NH}_{2}, \mathrm{OH}\right) .\end{array}$ & $\begin{array}{l}\left.\delta 6.13 \text { (brs, } 2 \mathrm{H}, \mathrm{NH}_{2}\right), \delta 8.1-7.01(\mathrm{~m}, 11 \mathrm{H} \\
\text { aromatic protons). }\end{array}$ \\
\hline $11 b$ & $\begin{array}{l}1655-1715(3 \mathrm{C}=\mathrm{O}), 3100-3450 \\
\left(\mathrm{NH}_{2}, \mathrm{OH}\right) .\end{array}$ & $\begin{array}{l}\delta 5.99(\text { brs, } 2 \mathrm{H}, \mathrm{NH} 2), \delta 8.1-7.01(\mathrm{~m}, 11 \mathrm{H} \\
\text { aromatic protons). }\end{array}$ \\
\hline $11 \mathrm{c}$ & $\begin{array}{l}1660-1715(3 \mathrm{C}=\mathrm{O}), 3100-3400 \\
\left(\mathrm{NH}_{2}\right) \text {. }\end{array}$ & $\begin{array}{l}\delta 1.25\left(\mathrm{~s}, 6 \mathrm{H}, 2 \mathrm{CH}_{3}\right), \delta 6.12\left(\mathrm{brs}, 2 \mathrm{H}, \mathrm{NH}_{2}\right), \delta \\
8.1-7.01(\mathrm{~m}, 8 \mathrm{H} \text { aromatic protons). }\end{array}$ \\
\hline
\end{tabular}


Table II (Continued)

\begin{tabular}{|c|c|c|}
\hline Compound No. & IR $v_{\max } / \mathrm{cm}^{-1}$ & ${ }^{1}$ H-NMR (DMSO) ppm \\
\hline $12 \mathrm{a}$ & $\begin{array}{l}1665-1720(3 \mathrm{C}=\mathrm{O}), 3100-3450 \\
(\mathrm{NH} 2, \mathrm{OH}) .\end{array}$ & $\begin{array}{l}\delta 2.53\left(\mathrm{~s}, 2 \mathrm{H}, \mathrm{CH}_{2} \text { of thiazolidinone), } \delta\right. \\
\left.5.95 \text { (brs, } 2 \mathrm{H}, \mathrm{NH}_{2}\right), \delta 8.1-7.01(\mathrm{~m}, 10 \mathrm{H} \\
\text { aromatic protons). }\end{array}$ \\
\hline $12 b$ & $\begin{array}{l}1660-1725(3 \mathrm{c}=\mathrm{O}), 3100-3450 \\
\left(\mathrm{NH}_{2}, \mathrm{OH}\right)\end{array}$ & $\begin{array}{l}\delta 2.55\left(\mathrm{~s}, 2 \mathrm{H}, \mathrm{CH}_{2} \text { of thiazolidinone), } \delta\right. \\
\left.6.01 \text { (brs, } 2 \mathrm{H}, \mathrm{NH}_{2}\right), \delta 8.1-7.01(\mathrm{~m}, 10 \mathrm{H}, \\
\text { aromatic protons). }\end{array}$ \\
\hline $12 \mathrm{c}$ & $\begin{array}{l}1655-1720(3 \mathrm{C}=\mathrm{O}), 3100-3400 \\
\left(\mathrm{NH}_{2}\right) .\end{array}$ & $\begin{array}{l}\delta 1.22\left(\mathrm{~s}, 6 \mathrm{H}, 2 \mathrm{CH}_{3}\right), \delta 2.51\left(\mathrm{~s}, 2 \mathrm{H}, \mathrm{CH}_{2}\right. \\
\text { of thiazolidinone), } \delta 5.98\left(\text { brs, } 2 \mathrm{H}, \mathrm{NH}_{2}\right) \text {, } \\
\delta 8.1-7.01 \text { (m, } 7 \mathrm{H} \text { aromatic protons). }\end{array}$ \\
\hline $13 \mathrm{a}$ & $\begin{array}{l}1650-1715(2 \mathrm{C}=\mathrm{O}), 3100-3400 \\
\left(\mathrm{NH}_{2}\right) .\end{array}$ & $\begin{array}{l}\left.\delta 6.55 \text { (brs, } 2 \mathrm{H}, \mathrm{NH}_{2}\right), \delta 8.1-7.01(\mathrm{~m}, 9 \mathrm{H}, \\
\text { aromatic protons). }\end{array}$ \\
\hline $13 b$ & $\begin{array}{l}1655-1718(2 \mathrm{C}=\mathrm{O}), 3100-3450 \\
\left(\mathrm{NH}_{2}, \mathrm{OH}\right) .\end{array}$ & $\begin{array}{l}\left.\delta 6.65 \text { (brs, } 2 \mathrm{H}, \mathrm{NH}_{2}\right), \delta 8.1-7.01(\mathrm{~m}, 9 \mathrm{H} \\
\text { aromatic protons). }\end{array}$ \\
\hline $13 \mathrm{c}$ & $\begin{array}{l}1665-1725(2 \mathrm{C}=\mathrm{O}), 3100-3400 \\
\left(\mathrm{NH}_{2}\right) .\end{array}$ & $\begin{array}{l}\delta 6.75\left(\mathrm{brs}, 2 \mathrm{H}, \mathrm{NH}_{2}\right), \delta 8.1-7.01(\mathrm{~m}, 8 \mathrm{H}, \\
\text { aromatic protons). }\end{array}$ \\
\hline $14 \mathrm{a}$ & $\begin{array}{l}1670-1725(2 \mathrm{C}=\mathrm{O}), 3100-3400 \\
\left(\mathrm{NH}_{2}\right) .\end{array}$ & $\begin{array}{l}\delta 3.31(\mathrm{~s}, 3 \mathrm{H}, \mathrm{COCH} 3), \delta 6.77 \text { (brs, } 2 \mathrm{H}, \\
\mathrm{NH} 2), 8.1-7.01(\mathrm{~m}, 8 \mathrm{H}, \text { aromatic protons). }\end{array}$ \\
\hline $14 \mathrm{~b}$ & $\begin{array}{l}1669-1720(2 \mathrm{C}=\mathrm{O}), 3100-3450 \\
\left(\mathrm{NH}_{2}, \mathrm{OH}\right) .\end{array}$ & $\begin{array}{l}\delta 3.30\left(\mathrm{~s}, 3 \mathrm{H}, \mathrm{COCH}_{3}\right), \delta 6.74 \text { (brs, } 2 \mathrm{H} \\
\left.\mathrm{NH}_{2}\right), 8.1-7.01(\mathrm{~m}, 8 \mathrm{H} \text { aromatic protons). }\end{array}$ \\
\hline $14 \mathrm{c}$ & $\begin{array}{l}1675-1725(2 \mathrm{C}=\mathrm{O}), 3100-3450 \\
\left(\mathrm{NH}_{2}\right) .\end{array}$ & $\begin{array}{l}\delta 3.35\left(\mathrm{~s}, 3 \mathrm{H}, \mathrm{COCH}_{3}\right), \delta 6.79 \text { (brs, } 2 \mathrm{H}, \\
\left.\mathrm{NH}_{2}\right), 8.1-7.01(\mathrm{~m}, 7 \mathrm{H}, \text { aromatic protons). }\end{array}$ \\
\hline $15 \mathrm{a}$ & $1685(\mathrm{C}=\mathrm{O}), 3100-3400\left(\mathrm{NH}_{2}\right)$. & $\begin{array}{l}\delta 6.58\left(\mathrm{brs}, 2 \mathrm{H}, \mathrm{NH}_{2}\right), \delta 8.1-7.01(\mathrm{~m}, 13 \mathrm{H}, \\
\text { aromatic protons). }\end{array}$ \\
\hline $15 b$ & $\begin{array}{l}1690(\mathrm{C}=\mathrm{O}), 3100-3450\left(\mathrm{NH}_{2},\right. \\
\mathrm{OH}) .\end{array}$ & $\begin{array}{l}\delta 6.62\left(\mathrm{brs}, 2 \mathrm{H}, \mathrm{NH}_{2}\right), 8.1-7.01(\mathrm{~m}, 13 \mathrm{H}, \\
\text { aromatic protons). }\end{array}$ \\
\hline $15 \mathrm{c}$ & $1700(\mathrm{C}=\mathrm{O}), 3100-3400\left(\mathrm{NH}_{2}\right)$ & $\begin{array}{l}\delta 6.68\left(\mathrm{brs}, 2 \mathrm{H}, \mathrm{NH}_{2}\right), 8.1-7.01(\mathrm{~m}, 12 \mathrm{H}, \\
\text { aromatic protons). }\end{array}$ \\
\hline $16 \mathrm{a}$ & $1684(\mathrm{C}=\mathrm{O}), 3100-3400\left(\mathrm{NH}_{2}\right)$ & $\begin{array}{l}\left.\delta 6.54 \text { (brs, } 2 \mathrm{H}, \mathrm{NH}_{2}\right), 8.1-7.01(\mathrm{~m}, 8 \mathrm{H}, \\
\text { aromatic protons). }\end{array}$ \\
\hline $16 b$ & $\begin{array}{l}1689(\mathrm{C}=\mathrm{O}), 3100-3450\left(\mathrm{NH}_{2},\right. \\
\mathrm{OH}) .\end{array}$ & $\begin{array}{l}\left.\delta 6.56 \text { (brs, } 2 \mathrm{H}, \mathrm{NH}_{2}\right), 8.1-7.01(\mathrm{~m}, 8 \mathrm{H}, \\
\text { aromatic protons). }\end{array}$ \\
\hline $16 \mathrm{c}$ & $1695(\mathrm{C}=\mathrm{O}), 3100-3400\left(\mathrm{NH}_{2}\right)$ & $\begin{array}{l}\left.\delta 6.60 \text { (brs, } 2 \mathrm{H}, \mathrm{NH}_{2}\right), 8.1-7.01(\mathrm{~m}, 7 \mathrm{H}, \\
\text { aromatic protons). }\end{array}$ \\
\hline $17 \mathrm{a}$ & $\begin{array}{l}1685-1715(2 \mathrm{C}=\mathrm{O}), 3100-3400 \\
\left(\mathrm{NH}, \mathrm{NH}_{2}\right) .\end{array}$ & $\begin{array}{l}\left.\delta 6.1 \text { (brs, } 2 \mathrm{H}, \mathrm{NH}_{2}\right), 8.1-7.01(\mathrm{~m}, 8 \mathrm{H}, \\
\text { aromatic protons), } \delta 11.12(\mathrm{brs}, 1 \mathrm{H}, \mathrm{NH})\end{array}$ \\
\hline $17 \mathrm{~b}$ & $\begin{array}{l}1690-1720(2 \mathrm{C}=\mathrm{O}), 3100-3450 \\
\left(\mathrm{NH}, \mathrm{NH}_{2}, \mathrm{OH}\right) .\end{array}$ & $\begin{array}{l}\left.\delta 6.23 \text { (brs, } 2 \mathrm{H}, \mathrm{NH}_{2}\right), 8.1-7.01(\mathrm{~m}, 8 \mathrm{H}, \\
\text { aromatic protons), } \delta 11.2(\mathrm{brs}, 1 \mathrm{H}, \mathrm{NH}) .\end{array}$ \\
\hline $17 \mathrm{c}$ & $\begin{array}{l}1695-1725(2 \mathrm{C}=\mathrm{O}), 3100-3400 \\
\left(\mathrm{NH}, \mathrm{NH}_{2}\right) .\end{array}$ & $\begin{array}{l}\left.\delta 6.45 \text { (brs, } 2 \mathrm{H}, \mathrm{NH}_{2}\right), 8.1-7.01(\mathrm{~m}, 7 \mathrm{H}, \\
\text { aromatic protons), } \delta 11.35 \text { (brs, } 1 \mathrm{H}, \mathrm{NH})\end{array}$ \\
\hline
\end{tabular}


Table II (Continued).

\begin{tabular}{|c|l|l|}
\hline Compound No. & \multicolumn{1}{|c|}{ IR v $_{\text {max }} / \mathbf{c m}^{-1}$} & \multicolumn{1}{|c|}{${ }^{\mathbf{1}} \mathbf{H}-\mathbf{N M R}$ (DMSO) ppm } \\
\hline $18 \mathrm{a}$ & $\begin{array}{l}1686(\mathrm{C}=\mathrm{O}), 3100-3400(\mathrm{NH}, \\
\left.\mathrm{NH}_{2}\right) .\end{array}$ & $\begin{array}{l}\left.\delta 6.33 \text { (brs, 2H, } \mathrm{NH}_{2}\right), 8.1-7.01(\mathrm{~m}, 8 \mathrm{H}, \\
\text { aromatic protons), } \delta 10.95(\mathrm{brs}, 1 \mathrm{H}, \mathrm{NH}) .\end{array}$ \\
\hline $18 \mathrm{~b}$ & $\begin{array}{l}1689(\mathrm{C}=\mathrm{O}), 3100-3450\left(\mathrm{NH}, \mathrm{NH}_{2},\right. \\
\mathrm{OH}) .\end{array}$ & $\begin{array}{l}\delta 6.35\left(\mathrm{brs}, 2 \mathrm{H}, \mathrm{NH}_{2}\right), 8.1-7.01(\mathrm{~m}, 8 \mathrm{H}, \\
\text { aromatic protons), } \delta 10.98(\mathrm{brs}, 1 \mathrm{H}, \mathrm{NH}) .\end{array}$ \\
\hline $18 \mathrm{c}$ & $\begin{array}{l}1693(\mathrm{C}=\mathrm{O}), 3100-3400(\mathrm{NH}, \\
\left.\mathrm{NH}_{2}\right) .\end{array}$ & $\begin{array}{l}\delta 6.55\left(\text { brs, } 2 \mathrm{H}, \mathrm{NH}_{2}\right), 8.1-7.01(\mathrm{~m}, 7 \mathrm{H}, \\
\text { aromatic protons }), 11.02(\text { brs, } 1 \mathrm{H}, \mathrm{NH}) .\end{array}$ \\
\hline
\end{tabular}

\section{References}

[1] K. Haraguchi; Y. Kubota; H. Tanaka; J. Org. Chem. 69, 1831 (2004).

[2] M. Cushman; T. Sambaiah; G. Jin; B. Illarionov; M. Fischer; Bacher, A.J. Org. Chem. 69, 601 (2004).

[3] G. Depecker; N. Patino; C.D. Giorgio; R. Terreux; D. Cabrol-Bass; C. Bailly; A.M. Aubertin; R. Condom; Org. Biomol. Chem. 2,74 (2004).

[4] S.F. Wnuk; E. Lewandowska; D.R. Companioni; P.I. Garcia; Jr.; J.A. Secrist; Org. Biomol. Chem. 2,120 (2004).

[5] A. Bendich; P.J. Russell; J.J. Fox; J.Am. Chem. Soc. 76, 6073 (1954).

[6] S. Kobayashi; Chem. Pharm. Bull. 21, 941 (1973).

[7] A. Ali; G.E. Taylor; D.W. Graham; Wo 0129045, (2001).

[8] S.A. Armstrong; J.M. Berge; P. Brown; J.S. Elder; A.K. Forrest; D.W. Hamprecht; R.L. Jervest; Wo 0071524, (2000).

[9] E.R. El-Bendary; F.A. Badria; Arch. Pharm. 333, 99 (2000).

[10] A. Tetsuya; M. Shogo; I. Fumio; Y. Masuo; N. Masafuml; EP 0733633, (1996).

[11] B.A. Dumaitre; N. Dodic; EP 636636, (1994).

[12] S.F. Campbell; A.R. Mackenzie; A. Wood; Wo 16644, (1996).

[13] A.F. Burchat; D.J. Calderwood; M.M. Friedman; G.C. Hirst; B.H. Li; P. Rafferty; K. Ritter; B.S. Skinner; Bioorg. Med. Chem. Lett. 12, 1687 (2002).

[14] B. Shaikh; W.A. Moats; Liquid chromatographic analysis of antibacterial drug residues in food products of animal orgin, J. Chromatogar. 643, 369-378, (1993).

[15] S.F. Sundloff; J. Cooper; in W.A. Moats, M.B. Medina (Eds.), ACS Symposium Series 636: Veterinary Drug Residues(Symposium at the national mecting of the American Chemical Society, Anaheim, CA), American Chemical Society, Washington, DC, p. 5, (1995).

[16] N.A.A. El-Kanzi, H.A. Soliman, Phosphorus, Sulfur and Silicon and the related elements vol.183:1679 -1690 (2008).

[17] N.A.A. El-Kanzi; A.K. Khalafallah, and M. Younis Phosphorus, sulfur and silicon and the related elements Vol. 182, No. 5, $1163-1181,2007$. 


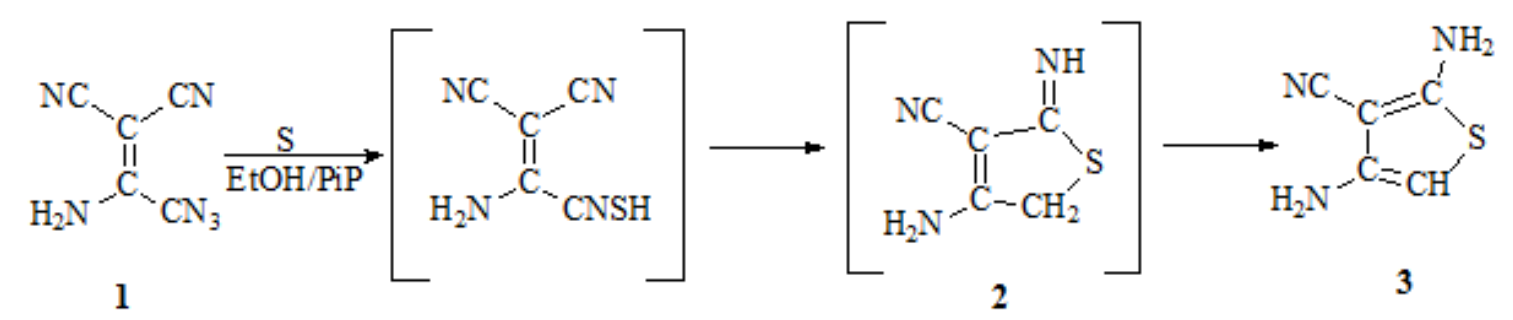

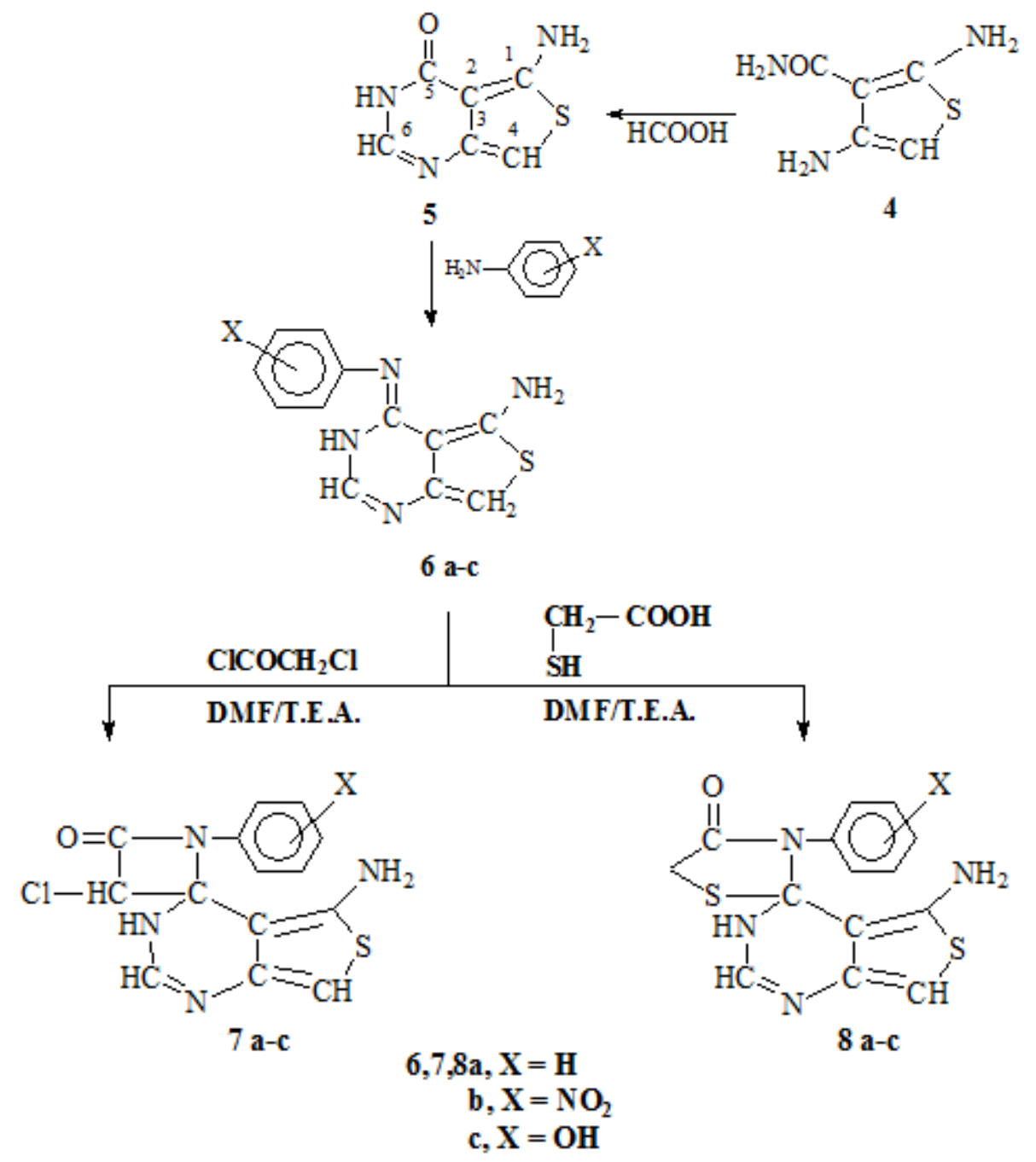

Scheme 1 
<smiles>CC(=O)OCC(=O)OCC(=O)OCC(=O)n1cnc2csc(N)c2c1=O</smiles>

5<smiles>Nc1scc2ncn(C(=O)C=N[Al])c(=O)c12</smiles>

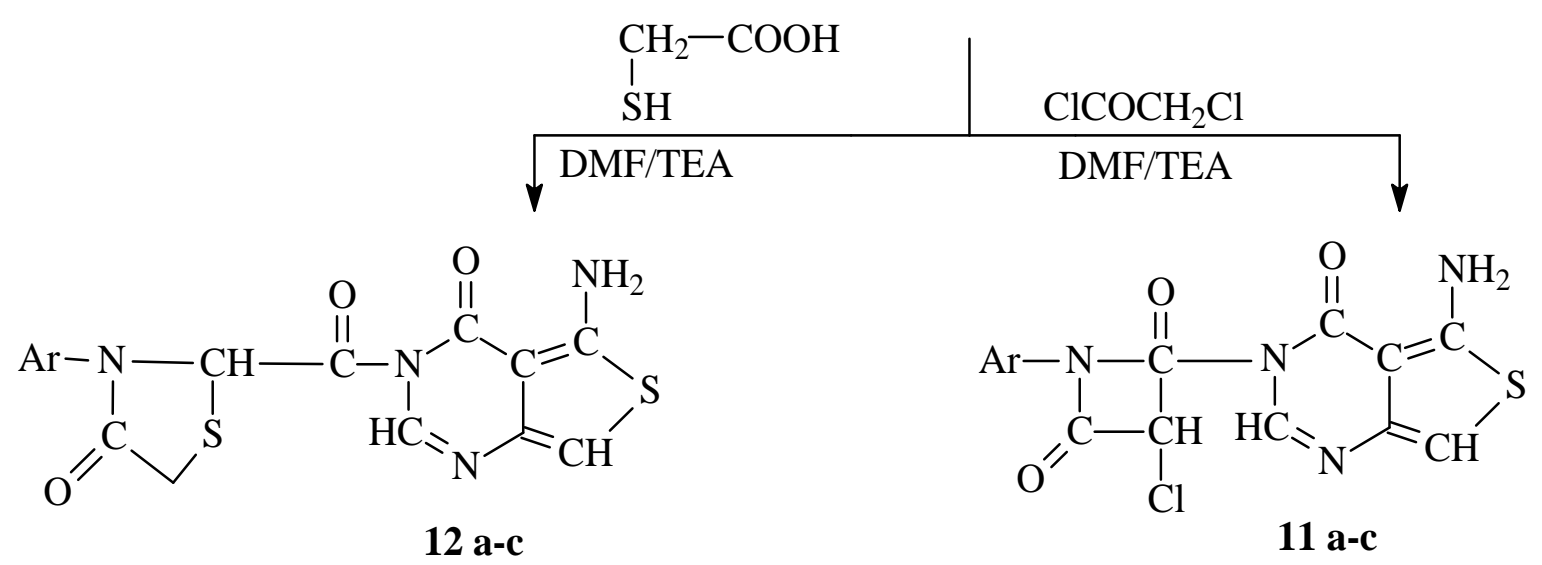

where a, Ar $\alpha$-nitroso $\beta$-naphthol; Ar $\beta$-nitroso $\alpha$-naphthol; c, Ar p-nitroso N,N-dimethylaniline

Scheme2 


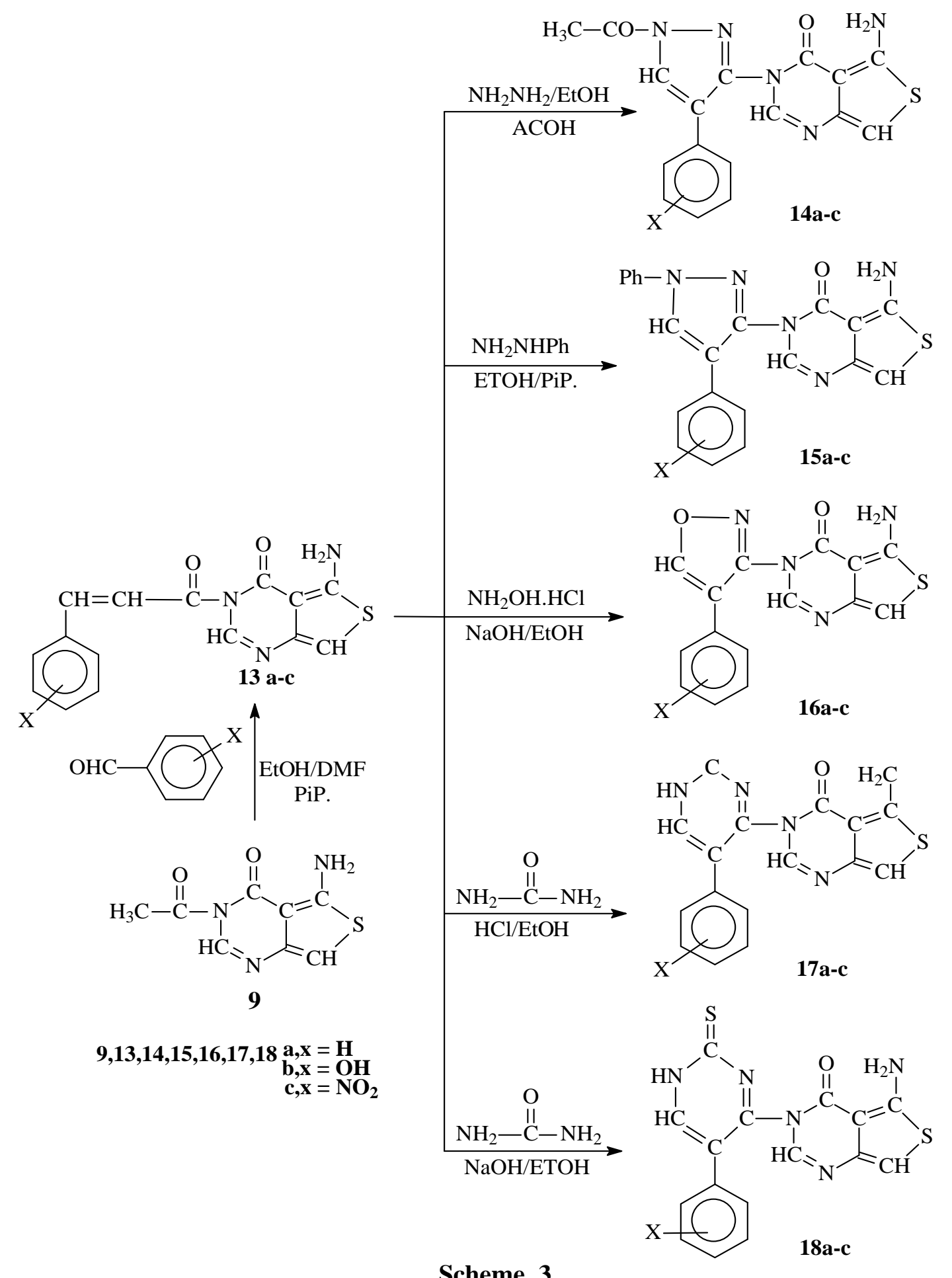

Scheme 3 